\title{
La langue française au Rwanda. Chronique d'une mort programmée
}

Obed Nkunzimana

University of New Brunswick

C'est un fait bien connu, l'Afrique incarne l'épine dorsale de la francophonie internationale. Mais depuis quelques années, une inquiétude plane. En 2009, le Rwanda, ancienne colonie belge et membre de la francophonie claque la porte et s'affilie au Commonwealth. Un peu plus tard, l'anglais devient officiellement la langue unique de formation du primaire à l'université, supplantant ainsi le français, considéré désormais comme un simple cours de langue seconde.

Au moment où les analystes tentent de décoder les vraies motivations de ce revirement unique et radical, retentit ce que certains appellent « un coup de tonnerre dans le ciel de la francophonie »(Couldiati) : autour du XIVe Sommet (Kinshasa, octobre 2012), le Gabon, souvent décrit comme la chasse gardée de la France et grand défenseur de la francophonie, annonce, par la voix de son Président même, la volonté de faire de l'anglais une deuxième langue officielle, en vantant le modèle du Rwanda, où il se rend pour une visite officielle.

Comme si cela n'était pas suffisant, le Burundi, quelques jours avant ce sommet, emboîte le pas en déposant sa demande d'adhésion au club anglophone. Il semblerait que l'Algérie et le Madagascar soient très intéressés par la communauté anglophone (Ouazani). Plus surprenant encore, un journaliste du Sénégal, pays de Senghor (feu membre de l'Académie française) et Abdou Diouf (Secrétaire général de l'Organisation internationale de la Francophonie) va même plus loin en affirmant radicalement que «La francophonie n'a plus de raison d'être sur le continent, et en particulier au Sénégal [...]. La langue anglaise doit s’imposer»(Thiam 2012). Décidément, comme le résume bien Glez, le caricaturiste franco-burkinabé, « en Afrique, la francophonie perd son latin » (2012).

Notre objectif n'est pas d'établir un diagnostic approfondi de cette "crise", encore moins d'esquisser des solutions globales ou des propositions de revitalisation linguistique, surtout qu'il existe en Afrique et ailleurs dans le monde autant de francophonies que d'espaces individuels, nationaux, régionaux et internationaux. Plutôt que d'en rester aux généralités africaines sur lesquelles nous ne disposons pas de données probantes, nous proposons 
de nous concentrer sur un cas particulier, le Rwanda. Nous tenterons de montrer, d'abord, en jetant un regard critique sur certains aspects de l'entreprise coloniale belge, notamment sa stratégie éducative, que le déclin du français, et peut-être un jour sa disparition comme langue d'usage à tous les niveaux, était programmé d'avance, inscrit non seulement dans les gènes mêmes de son implantation par l'autorité coloniale belge, il y a environ un siècle de cela, mais surtout dans la singularité du contexte farouchement monolingue du Rwanda traditionnel. Nous examinerons ensuite les conditions qui rendent le rebond sinon impossible, du moins improbable, car le chemin est parsemé d'obstacles presque infranchissables, en raison de la nouvelle donne géopolitique et linguistique du Rwanda et de sa politique économique axée sur les nouvelles technologies de l'information où l'anglais reste prédominant.

\section{De quelques éléments historiques et méthodologiques}

Concernant cette tendance inquiétante du déclin de la langue française en Afrique, soit dit en passant, certaines hypothèses d'explication qui sont avancées dépendent de la relation historique de proximité avec la France ou de méfiance à son égard. Par exemple, pour l'écrivaine- journaliste québécoise Denise Bombardier, dans Lettre ouverte à ces Français qui se pensent le nombril du monde, sorte de déclaration d'amour et de guerre adressée à une nation dont elle dit partager l'héritage historique et culturel, ce déclin serait une sorte de défection devant le « recul du prestige de la France auquel correspond la rétrogradation du français parlé [et] un recul pour toute la francophonie» (128). D'autres y voient une volonté de conjurer ce que le Camerounais Ambroise Kom appelle "la malédiction francophone », dans son essai intitulé La malédiction francophone. Défis culturels et condition postcoloniale en Afrique, c'est-àdire l'urgence de sortir du joug de l'ancien pouvoir colonial, la France, qui, selon lui, a souvent fait de la francophonie un instrument de ses propres ambitions impérialistes, plutôt qu'un levier de développement, d'épanouissement et de rapprochement des peuples.

Dans cet ordre d'idées, la Belgique n'échappe pas à la règle. À l'instar d'autres puissances coloniales dans leur ruée vers les espaces à exploiter, son objectif prioritaire était de mettre en place tout un dispositif visant à renforcer les assises du pouvoir colonial et accroître les possibilités d'expansion et d'exploitation. Ainsi, en examinant l'intention et les conditions d'instauration du français au Rwanda au début du siècle dernier, sans oublier l'impact de cet acte inaugural sur l'état actuel de la langue de Voltaire, c'est tout l'imaginaire colonial qui est interrogé ici. De cet imaginaire, l'on retiendra surtout ses stratégies de conquête et ses mécanismes d'aliénation et d'assujettissement, à

This work is licensed under a Creative Commons Attribution 3.0 License 
travers lesquels tout acte posé et tout projet initié par l'autorité coloniale se fait sous le signe de l'expropriation globale de l'Autre et de la politique de divide et impera qui inocule le virus de division et d'infériorité dans le corps social du pays conquis.

Nous évoquerons aussi la singularité historique rwandaise en matière linguistique qui n'aura pas facilité l'enracinement et la vulgarisation d'une nouvelle langue étrangère, en l'occurrence le français, qui se heurte à un monolinguisme nationaliste entretenu par une monarchie de droit divin imperméable aux valeurs extérieures.

Trois périodes-clés seront brièvement examinées ici : a) l'époque coloniale belge (1916-1962) dont tout le système éducatif, et l'enseignement du français en particulier, n'est qu'une stratégie de renforcement de l'emprise coloniale et de propagation de la doctrine catholique; b) la période des indépendances jusqu'au génocide de 1994(1962-1994) où le français connaît un essor fulgurant et acquiert un statut de prestige pour une élite dirigeante connectée au monde extérieur avec tous les privilèges réservés aux "happy few" et autres "has been" ; c) et, enfin, de 1994 à aujourd'hui, période marquée par une chute brutale du français, qui perd son statut de langue officielle et marginalisé au profit de la langue de Shakespeare.

\section{Le français dans le Rwanda colonial: un instrument de prédation et de division}

\section{Le français à l'époque coloniale belge}

L'introduction et l'enseignement de la langue française au Rwanda est, dès le départ, un terrain véritablement miné par ses initiateurs mêmes ; un projet mal parti, comportant les clauses de son propre inaboutissement et, de ce fait, les germes de sa future disparition. C'est que, dès les débuts de la colonisation belge au Rwanda (1916-1962), le français fut enseigné à un petit groupe d'élus, sélectionnés essentiellement dans la classe dirigeante tutsi et destinés à être des auxiliaires de l'administration coloniale comme futurs enseignants et interprètes, bref à être les yeux et les oreilles des autorités belges (Kabanza; Munyankesha). S'amorce, dès lors, un dangereux processus de distinction préférentielle, c'est-à-dire de distorsion du clivage existant hutu/tutsi qui incorpore, désormais, le virus de la préférence et de la hiérarchisation entre ces deux ethnies principales, d'une part, et au sein de l'ethnie dirigeante tutsi, d'autre part, étant donné que tous les Tutsis n'avaient pas accès à ce privilège colonial. 
On introduit donc un nouveau critère de valeur et de prestige dans le jugement et la classification des gens : il y a désormais une petite minorité, le cercle restreint des locuteurs civilisés, souvent appelés " des évolués » qui parlent la langue bénie des Blancs et les masses indigènes parlant un dialecte sauvage. Dès lors, tout individu ayant le privilège d'apprentissage du français se sent investi d'une supériorité hiérarchique et d'une mission quasi messianique, d'autant plus que l'enseignement était pris en charge par les prélats catholiques et protestants. Aux distinctions et classifications ethniques jadis faites essentiellement sur des bases socioéconomiques, parfois sur l'origine géographique du territoire rwandais, s'ajoute un facteur supplémentaire de distinction et de division qui ne fait que renforcer l'emprise du pouvoir colonial sur la population.

Comme si ce critère de zizanie n'était pas suffisant, même cette minorité de prétendus «évolués » est loin d’être épargnée par le préjugé colonial et la stratégie impérialiste. En référence au propos du prêtre belge Lacger sur l'essence de la stratégie pédagogique coloniale belge, le linguiste rwandais Kabanza (27) rappelle que la matière et la méthode d'enseignement $\mathrm{du}$ français doivent rester minimalistes et répétitives pour mieux les adapter au piètre niveau cognitif de l'indigène : «Selon les instructions officielles, on doit, autant que possible, ralentir le processus d'apprentissage chez le Noir en fonction de ses mécanismes de fonctionnement cérébral! Son processus de pensée est exceptionnellement lent, et l'est davantage son activité mentale. Il faut alors mettre tout au ralenti, les résultats les plus heureux sont escomptés 'mais exigent de plus longs délais' (Lacger, L. de 591) ».

Selon l'auteur de l'article, s'ajoutent à ce cliché raciste des méthodes inadaptées, le manque de matériel didactique, les missionnaires qui s'improvisent enseignants non de la langue française, mais de la morale catholique (souvent traduite en Kinyarwanda) et de la propagande coloniale, sans oublier les conflits Wallons/Flamands de la métropole qui s'invitent dans la colonie et fragilisent davantage une situation déjà précaire. Ce qui explique pourquoi la langue française est, dès le départ, un projet moribond, sans avenir, étouffé par le calcul égocentrique de ses propres promoteurs.

Rappelons que derrière cette stratégie du ralenti et du minimum se cache un autre plan inavoué, mais réel, lié à la férocité de la concurrence des puissances coloniales : en ce qui concerne le Congo belge, par exemple, on parle carrément du « refus de l'administration coloniale belge d'encourager et de généraliser l'enseignement du français » (Kasende), et ce, pour lui couper tout contact extérieur, afin de rester seul maitre à bord. Par ailleurs, selon Ndaywel è Nziem, en vue de garder intact le statut de supériorité paternaliste 
du colonisateur sur le colonisé, la maîtrise du français par ce dernier « devait être évitée autant que possible, car elle était source d'orgueil et de prétention » (ibid.).

Tous ces calculs et ces cachotteries qui émaillent les débuts d'enseignement de la langue française lors de la période coloniale vont avoir un impact désastreux sur les efforts ultérieurs consentis à son renforcement et son développement dans la période postcoloniale. Mais plus déterminant encore dans ces ratés d'une véritable implantation de la langue de Voltaire, est la particularité linguistique de ce petit pays dit «des milles et une collines » (ou plutôt «mille et un sourires », selon certains). Contrairement à tous les autres pays du continent, le Rwanda est le rare pays (à part le Burundi), à parler une seule et même langue qui s'appelle le Kinyarwanda, mot qui signifie plus qu'une langue: une manière d'être et de faire à la rwandaise, une exception rwandaise ${ }^{1}$. Fort de sa richesse lexicale et de son prestige historique, de sa sophistication grammaticale et structurale, de sa poésie pastorale et guerrière ainsi que de ses saveurs et accents régionaux, le kinyarwanda a toujours été considéré par les Rwandais comme une langue de fierté et de musicalité, permettant aux locuteurs de communiquer et de traduire la réalité avec des niveaux de langage ou registres adéquats, sans aucune nécessité de recours à d'autres langues ou autres emprunts langagiers.

Ainsi, le Kinyarwanda a tous «les attributs (autonomie, vitalité, historicité, standardisation) qui fondent le prestige d'une langue selon la typologie de Stewart. [...] Le kinyarwanda est utilisé dans des situations formelles, publiques et officielles et n'est [pas] simplement la langue des échanges privés et familiaux. Son prestige est historique; la parenthèse coloniale (environ un demi-siècle) ne l'a pas entamé » (Jouannet 23).

Bref, l'introduction manquée du français n'est pas seulement due à la mauvaise foi et la mesquinerie coloniales, elle est aussi, bien que dans une moindre mesure, tributaire d'un contexte culturel et linguistique fermé, autosuffisant et donc imperméable à une autre langue, une langue d'autant plus indigeste qu'elle est enfoncée de force dans la gorge, alors qu'elle n'est ni outil d'interactions dans la vie quotidienne ni instrument de cohésion nationale, comme ce fut le cas au Congo par exemple. Ces deux facteurs, l'un endogène

${ }^{1}$ Ce n'est d'ailleurs pas pour rien que le mot 'Rwanda' recèle une connotation d'immensité et de grandeur, malgré l'exiguiité géographique du pays. Pour les Rwandais, leur pays est une terre bénie des dieux, voire la demeure des dieux, d'où le dicton : « Dieu passe la journée ailleurs et rentre le soir chez-lui au Rwanda' (Imana yirinw'ahandi igatab'i Rwanda). Cet imaginaire collectif de prestige identitaire et linguistique est, à notre avis, un autre obstacle majeur à l'enracinement d'une langue étrangère au Rwanda.

This work is licensed under a Creative Commons Attribution 3.0 License 
et l'autre exogène, vont sceller, lors des périodes subséquentes, le destin fragile de la langue française au Rwanda.

\section{Le français à l'ère postcoloniale}

À l'époque postcoloniale (au sens historique), on peut parler ici de deux périodes déterminantes dans l'essor de la langue française: des indépendances en 1962 à la tragédie de 1994, période de consolidation et de stabilisation, malgré les aléas conjoncturels et les décisions politiques défavorables; et de 1994 à aujourd'hui, où le français subit une fin brutale à la surprise de tout le monde, se faisant remplacer manu militari par l'anglais.

De 1962-1994 (des indépendances au génocide de 1994) - Une consolidation fragile, mais prometteuse

Nous n'allons pas nous étendre sur cette période, car d'autres ont tenté de schématiser les moments-clés de cette histoire. Ntakirutimana (2010), par exemple, pour ne citer qu'un seul spécialiste, en dénombre quatre : «De 1961 à 1979 : Français, langue de prédilection»; «1979 à 1991 : Africanisation linguistique (kinyarwanda et swahili) »; «1991 à 1994 : le français accélère son développement » et enfin «1994 à nos jours: le français bat en retraite, l'anglais passe à la vitesse supérieure ».

L'idée principale qui se dégage de cette périodisation est que pendant toute cette période de trois décennies allant des indépendances jusqu'en 1994, le français se consolide, se stabilise et devient une langue de prestige, mais tout n'est pas joué pour autant, car ce développement très prometteur s'avère toujours précaire. Hormis les carences chroniques en matériels adaptés au contexte rwandais et en ressources humaines bien formées et compétentes, le français est fragilisé par deux forces corrosives, l'une historique, l'autre politique. Premièrement, la politique d'enseignement élitiste et minimaliste de la période coloniale pèse encore lourd, en ce sens que la langue française, dans de nombreux cas, est très peu maitrisée à l'oral comme à l'écrit. Deuxièmement, il reste une sorte de prothèse de luxe et occasionnelle, un trophée de prestige dont bénéficie une petite minorité de nouveaux intellectuels et fonctionnaires de la postcolonie ${ }^{2}$, héritiers de l'école coloniale $\mathrm{du}$ minimum et du mépris, misant sur la pédagogie du ralenti et du paternalisme clérical.

\footnotetext{
2 Terme utilisé par l'écrivain djiboutien Abdourahman Waberi qui constate des cicatrices indélébiles de la colonisation dans les nations et chez les peuples décolonisés.
} 
Par ailleurs, le nationalisme linguistique qui balaie l'Afrique dans les années 1970 et 1980 n'épargne pas le Rwanda, et ce, aux dépens du français dont l'état est déjà fragilisé. Vers la fin des années 1970, une réforme scolaire d'envergure est envisagée ayant comme objectif principal «la ruralisation [au primaire] et la mandisation des contenus d'enseignement à tous les niveaux [...]. Le développement du Kinyarwanda comme objet et langue d'enseignement » (Gakuba 6 ; l'auteur souligne). Ce processus de revalorisation de la langue nationale, le kinyarwanda (similaire aux initiatives concernant le swahili), dans le système éducatif ${ }^{3}$ fera long feu suite aux critiques exprimées notamment par les parents qui n'y voient aucun avantage professionnel et intellectuel, et à cause de l'absence des moyens de sa mise en application. Ainsi, bien que légitime et louable dans ses intentions, la réforme essuiera une double défaite : non seulement elle marque un recul important dans l'utilisation et la promotion de la langue française, qui est restée un outil de prestige et d'ouverture internationale, mais elle n'a pas non plus servi à promouvoir davantage la langue et les valeurs rwandaises.

Mais, malgré cette parenthèse idéologique contestée et improductive, la langue de Voltaire s'affirme comme médium officiel dans les écoles, l'administration publique et les relations internationales et reste un outil d'ascension sociale incontournable. On peut même dire que cette période est, en quelque sorte, l'âge d'or de la langue française, d'autant plus que le gouvernement rwandais, en bon élève de l'Occident comme certains autres pays francophones africains, connaît un relatif renforcement du fait francophone notamment grâce à la coopération accrue avec la France, la Belgique, la Suisse romande et le Québec qui accueillent des boursiers rwandais et envoient des coopérants au pays. Les évènements de 1994 vont complètement modifier le tableau de la politique linguistique, entre autres profonds bouleversements.

\section{De 1995 à aujourd'hui ou quand le prestige change de camp}

L'emprise sur les hautes sphères du pouvoir politique et économique par les anciens réfugiés rwandais rentrés des pays anglophones limitrophes (Kenya, Tanzanie, Uganda) et du monde anglo-saxon (Grande-Bretagne, É.U.) va déterminer la suite des choses. De 1996 à 2008, le Rwanda est officiellement trilingue puisque l'on y parle le Kinyarwanda, le français, et

3 Ce retour aux sources est une tendance panafricaine : par exemple en République Démocratique du Congo, alors le Zaïre, on parle de «zaïrianisation », où l'on veut aller aussi loin que refuser non seulement les langues coloniales, mais aussi les prénoms propres à consonance occidentale (Jean, Paul, etc.,) ainsi que leurs accoutrements tels les costumes avec cravates, pour ne citer que ces exemples. 
l'anglais. Aux niveaux secondaire et postsecondaire, chaque personne peut choisir la langue d'enseignement qui lui convient selon ses compétences linguistiques. Mais ce système idéal n'allait pas durer, l'état ne disposant simplement pas des moyens nécessaires à son financement. Dans ce cas, pourquoi le Rwanda, qui se veut le meneur régional en matière de développement socioéconomique et d'exemplarité politique, n'opterait-il pas pour un bilinguisme fort, notamment pour maintenir des liens avec l'Afrique francophone? En réponse à la journaliste Jenny Costes, dans un article intitulé "Le français, un luxe inutile au Rwanda», un ministre rwandais explique: «Nous avons un lien historique avec le français, c'est vrai. Mais ce n'est pas rentable. On opterait bien pour une éducation bilingue, mais le Rwanda ne peut se payer le luxe de faire évoluer les deux langues parallèlement » (Le Devoir).

C'est donc ainsi qu'en octobre 2008 fut promulguée la loi sur l'exclusivité de l'anglais à tous les niveaux d'enseignement, du primaire à l'université. Entre temps, en 2009, le Rwanda, après des années de tractations et de coulisses diplomatiques, adhère au club anglophone du Commonwealth avec de nouvelles allégeances, la Grande-Bretagne et les États-Unis essentiellement, sans oublier le resserrement des liens avec les pays anglophones africains de la Communauté est-africaine et de l'Afrique australe dont l'Afrique du Sud principalement.

Il serait prétentieux, dans le cadre restreint de cet article, de tenter de cerner les motivations politiques ou stratégiques de ce revirement spectaculaire et unique en Afrique, et peut-être dans le monde. Nous laissons la question aux spécialistes des sciences politiques pour un regard plus approfondi sur cet acte sans précédent comme sur ses multiples conséquences. Notre objectif est plutôt de montrer que toute stratégie de revitalisation ou tout rêve de renaissance de la langue française est un pari difficile, un défi presque irréalisable et qu'il y a raison de désespérer quant à la survie du français au Rwanda. Ce qui constitue, à notre avis, l'arme la plus fatale à la langue de Voltaire, c'est que le prestige a changé de camp. Alors que le fait de parler français est devenu presque un fait de nostalgie, un acte de défi ou de résistance, voire un geste de survie pour certains, les expressions et les mots anglais prennent le dessus, émaillant le langage quotidien des Rwandais, surtout des jeunes adultes, y compris de ceux et celles qui n'en connaissent que quelques bribes. La langue du prestige socioprofessionnel et des contacts internationaux aujourd'hui au Rwanda, c'est l'anglais, et les francophones s'y prêtent tant bien que mal, par adhésion solidaire ou par la force des choses. Dans l'espace public, comme dans leurs conversations informelles, verbales ou 
électroniques, les mots anglais sont prédominants, en alternance avec le Kinyarwanda.

L'on pourrait saluer les efforts appréciables du Centre culturel français qui constitue probablement un dernier retranchement pour une possible reconquête d'un certain espace francophone dans lequel le français survivrait, faute de recouvrer son éclat et son prestige d'antan, mais l'espoir me semble minime quant à la survivance du français. À l'image de son emplacement dans un «coin» (French Corner $^{4}$ ) au sein de la bibliothèque publique, l'espace francophone survivra difficilement au rouleau compresseur de l'histoire, car le temps joue contre lui, même si rien n’est jamais irrémédiablement perdu.

\section{Conclusion}

C'est donc la chronique d'un déclin programmé d'avance. Après l'étau colonial qui fragilise ses fondements et limite délibérément sa portée, c'est la violence de l'histoire récente qui l'étouffe, le rendant presque invisible et inaudible, exactement comme au début de la colonisation où le français n'est rien qu'un instrument de domination et d'expropriation, plutôt qu'un nouveau produit de consommation recherché par une grande majorité de la population. Ce ne serait donc pas faux de dire que parler de francophonie dans ce pays fut une forme d'imposture flagrante, car le Rwanda n'a jamais véritablement été un pays francophone. Même avant l'imposition de l'anglais, il existe seulement $3 \%$ de «francophones réels » (expression orale et écrite convenable), selon le recensement de 2003 (Têtu : 335).

On rappellera, par ailleurs, que le français ne fut pas la première langue étrangère introduite au Rwanda. Avant les Belges, les Allemands avaient propagé le swahili, en vue d'intégrer ce petit pays dans ce qui s'appelait le «Deutsch Ost-Afrika » ou l'Afrique de l'Est-Allemande. Nous pourrions donc dire que la langue française fut un arbre exotique, prestigieux certes, mais sans

\footnotetext{
${ }^{4}$ Tout n'est pas encore perdu, car un dernier retranchement existe : dans les locaux actuels de " Rwanda Public Library » - la bibliothèque publique de Kigali, l'Institut français au Rwanda a obtenu un espace francophone nommé- et le nom est révélateur- « Le French Corner »- le coin francophone où les abonnés peuvent consulter des livres en français et d'autres ressources numériques. L'institut en question a adopté, entre autres, une approche extramurale qu'il appelle justement " hors les murs ». Grâce à ce qu'on appelle « Cellule mobile d'animations culturelles », l'Institut Français « coordonne régulièrement à travers le Rwanda des actions autour du livre, du conte, du cinéma (cinéma mobile) et des ateliers de formation informatique (ordinateurs portables, iPad). Il s'agit ici encore d'aller à la rencontre d'un public n'ayant pas accès à la culture » (Institut français du Rwanda).
}

This work is licensed under a $\underline{\text { Creative Commons Attribution 3.0 License }}$ 
racines profondes dans le terroir rwandais, dans le quotidien des gens ${ }^{5}$. Une sorte de météorite sans envergure, projeté d'une autre planète sur une surface restreinte et fermée de l'espace sociolinguistique rwandais, loin des nappes phréatiques de la culture locale et de l'imaginaire collectif.

L'on comprend pourquoi le changement de langue officielle ne fera pas longtemps verser de larmes au Rwanda, malgré le casse-tête administratif et scolaire qu'il continue de générer, ainsi que la grogne sourde d'une minorité de francophones qui, ne parlant pas anglais, doit mettre les bouchées doubles pour s'adapter à la nouvelle dynamique linguistique et politique. De plus, l'on n'oubliera pas que tout changement de langue est aussi un changement de repères et de réseaux socioprofessionnels, ce qui ne va pas sans anxiété et incertitudes.

Il n'empêche qu'une page a été tournée. Après une naissance tronquée et une croissance compliquée et incertaine, le français survivra mal aux soubresauts de son histoire parsemée de hauts et de bas. Son introduction, minée d'intentions malicieuses et divisionnistes, était vouée à l'échec par une politique coloniale d'enseignement sélectif fait à petite échelle et à très faible dose, alors que son développement ultérieur s'est fait en dent de scie et en vase clos élitiste sans enracinement populaire.

Les plus optimistes trouveront un leur réconfort dans la phrase de Philippe de Saint Robert qui, dans Lettre ouverte à ceux qui en perdent leur français, rappelle avec le poète mauricien Edouard Maunick que «La francophonie se fera malgré la France » (146). Malheureusement, un tel optimisme n'est pas de mise en ce qui concerne le Rwanda où la langue française est intimement associée à la France, du moins à une certaine France, qui est accusée d'avoir entretenu des liens directs et répréhensibles avec l'ancien régime génocidaire.

Rappelons par ailleurs que, dans les ex-colonies, toute langue seconde, peu importe laquelle, en tant qu'instrument de prestige, facteur d'ascension sociale et de pouvoir économique restera source inévitable d'inégalités et de divisions, et l'anglais, qui compte aussi un peu plus de 3\% de locuteurs Rwandais, n'échappe pas à cette règle. Mais il n'est pas moins vrai que, d'un

\footnotetext{
${ }^{5}$ Les littératures africaines regorgent d'exemples de transferts linguistiques entre les langues locales et la langue des colonisateurs, ce qui a donné ce qu’on nomme « le français petit nègre » en Afrique francophonie ou " le pidgin » en Afrique anglophone. Mais au Rwanda, la langue du colon, malgré la force imposée ses idéologèmes et ses codes culturels, a laissé, à ma connaissance, très peu de traces dans le kinyarwanda, à l'instar de l'allemand, le swahili et l'arabe.
}

This work is licensed under a $\underline{\text { Creative Commons Attribution 3.0 License }}$ 
point de vue géopolitique et stratégique, l'anglais s'avère un meilleur outil d'intégration régionale et internationale pour le Rwanda.

Le retour symbolique de la France pourrait-il changer la donne? Rien de moins sûr. Il ne pourra que prolonger son agonie, en le gardant sous respiration artificielle, car, quoi qu'il arrive, le nouvel ordre politico-linguistique est irréversible et, comme nous l'avons signalé, l'adversaire le plus coriace de la langue française, c'est cette forme de désaffiliation affective à son égard et de reconnexion à l'ailleurs dont font preuve la majorité des francophones qui, rappelons-le, n'ont besoin d'aucune autre langue étrangère pour communiquer entre eux. Par-dessus tout, la population rwandaise, surtout la jeunesse, se trouve exposée, parfois malgré elle, à l'anglais, dicté par l'omniprésence des médias sociaux et des nouvelles technologies de l'information, qui constituent le cheval de bataille des politiques de développement du gouvernement actuel.

Malgré tout, comme nous le savons, une langue apprise ne peut disparaître complètement, car subsistent toujours des poches de résistance et des îlots de survivance: des regroupements de parents francophones, conscients des avantages du bilinguisme, s'organisent pour fonder et gérer des écoles francophones, alors que ceux qui ont des moyens envoient leurs enfants dans les écoles française et belge à Kigali, ou en Europe et au Québec, malgré le poids financier que cela implique. On l'aura compris, ces tentatives de sauvetage de l'héritage francophone, loin d'être motivées par toute forme de militantisme linguistique, visent à fournir à certains jeunes des outils supplémentaires qui accroitraient leurs chances de mobilité et de promotion professionnelles. Peut-être la survie de la langue de Molière dépendra-t-elle de ce genre d'initiatives privées et de pragmatismes calculateurs d'une certaine frange de la société civile qui n'attend plus de salut dans des politiques officielles en la matière. 


\section{Bibliographie}

Addamah, Steven. " Burundi: Bujumbura demande d'adhérer au Commonwealth. " Le Griot.info. Quotidien panafricain d'informations générales. 5 Oct. 2012. Web. 12 Jan. 2014.

Bombardier, Denise. Lettre ouverte à ces Français qui se croient le nombril du monde. Paris : Albin Michel, 2000.

Costes, Fanny. "Le français, un luxe inutile au Rwanda. Le Devoir. 14 août. 2010. Web. 10 janvier 2014

Couldiati, Boulkindi. "L'Anglais au Gabon. Coup de tonnerre dans le ciel de la francophonie. » Jeune Afrique. 3 octobre 2012. Web. 6 mai 2014.

De Saint Robert, Philippe. Lettre ouverte à ceux qui en perdent leur français. Paris : Albin Michel, 1986.

Gakuba, Emmanuel. Les centres d'éducation rural et artisanal intégré (CERAI) au Rwanda: problèmes et perspectives. Paris: Institut international de planification de l'éducation, 1991. 1-56. Web. 14 janvier 2014.

Glez, Damien. «En Afrique la francophonie perd son latin ». 11 octobre 2012. Web. 29 avril 2014.

Jouannet, D. Francis. Le français au Rwanda: enquête lexicale. Paris : GERLASELAF, 1984.

Kabanza, Faustin. "L'enseignement du français à l'école coloniale du Rwanda. » L'enseignement du français en colonies : expériences inaugurales dans l'enseignement primaire. Ed. Morsly, Dalila. Paris : L’Harmattan, 2010. 1934.

Kasende, L.A. Jean-Christophe. "L'émergence de la conscience francophone au Congo-Kinshasa. » Documents pour l'histoire du français langue étrangère ou seconde 40-41 (2008). Web. 15 janvier 2014.

Kom, Ambroise. La malédiction francophone. Défis culturels et condition postcoloniale en Afrique. Hamburg/Yaoundé : LIT / CLÉ, 2000.

Molsly, Dalila, ed. L'enseignement du français en colonies: expériences inaugurales dans l'enseignement. Paris : L'Harmattan, 2010.

Munyankesha, Pascal. «Quel avenir pour le français dans la nouvelle politique linguistique du Rwanda?» Les Cabiers du GRELCE. 2 mai 2010. Web. 10 mai 2014.

Ndaywel è Nziem, Isidore. L'histoire du Zaïre. De l'héritage ancien à l'âge contemporain. Louvain-La-Neuve: Duculot, 1997.

Ntakirutimana, Évariste. «Le français au Rwanda.» Le Français en Afrique : Revue du Réseau des Observatoires du Français Contemporain en Afrique. 25 (2010): 19-31. Web. 5 mai 2014.

Ouazani, Cherif. « Rwanda Inc. De Voltaire à Shakespeare. » Jeune Afrique. 15 décembre 2009. Web. 6 mai 2014. 
Têtu, Michel. La Francophonie. Histoire, problématique et perspectives. Montréal : Guérin littérature, 1987.

Thiam, S. Pape. "L'Anglais doit s'imposer. » Le courrier international. 18 Oct. 2012. Web. 11 janvier 2014.

Williame, Jean-Claude. Les Belges au Rwanda, le parcours de la honte. Bruxelles : GRPI/Complexe, 1997. 